\title{
The Łojasiewicz Exponent of Semiquasihomogeneous Singularities ${ }^{* \dagger}$
}

\author{
SZYMON BRZOSTOWS KI
}

November 8, 2018

\begin{abstract}
Let $f:\left(\mathbb{C}^{n}, 0\right) \rightarrow(\mathbb{C}, 0)$ be a semiquasihomogeneous function. We give a formula for the local Łojasiewicz exponent $\mathscr{L}_{0}(f)$ of $f$, in terms of weights of $f$. In particular, in the case of a quasihomogeneous isolated singularity $f$, we generalize a formula for $\mathscr{L}_{0}(f)$ of KR A S IN S K I, O LE K S I K and PŁO S K I ([KOP09]) from 3 to $n$ dimensions. This was previously announced in [TYZ10], but as a matter of fact it has not been proved correctly there, as noticed by the AMS reviewer T. KRASIN S KI.

As a consequence of our result, we get that the Łojasiewicz exponent is invariant in topologically trivial families of singularities coming from a quasihomogeneous germ. This is an affirmative partial answer to Teissier's conjecture.
\end{abstract}

\section{Introduction}

The (local) Łojasiewicz exponent $l_{0}(f)$ of a holomorphic map-germ $f:\left(\mathbb{C}^{n}, 0\right) \rightarrow\left(\mathbb{C}^{m}, 0\right)$ is one of the possible generalizations of the order function from 1 to $n$ indeterminates. Namely, for $n=1$ the condition ord $f:=$ $\inf \left\{\operatorname{ord} f_{i}\right\}=k$ is equivalent to the condition

$$
C_{1}|z|^{k} \leqslant\|f(z)\| \leqslant C_{2}|z|^{k}
$$

for some positive constants $C_{1}, C_{2}$, in a neighbourhood of 0 . (Here $k<\infty$ exactly when $f \neq 0$ i.e. when $f$ is a finite mapping.) For $n>1$, the above condition splits: the optimal exponent in the right inequality (that is the biggest one) leads to the ordinary notion of the order ord $f$ of $f$ while the optimal exponent in the left inequality (that is the smallest one) is the Eojasiewicz exponent $l_{0}(f)$ of $f$. (Note that $l_{0}(f)<\infty$ exactly when $f$ is a finite mapping, unlike the order function.) To state it more formally,

$$
l_{0}(f):=\inf \left\{q \in \mathbb{Q}_{>0}: C_{1}\|z\|^{q} \leqslant\|f(z)\| \text {, for some } C_{1}>0 \text { and all }\|z\| \ll 1\right\} .
$$

The norms $\|\cdot\|$ in the definition are any convenient ones. An important (and, at first, maybe a little surprising) fact is that $l_{0}(f)$, if finite, is a positive rational number. Indeed, one can prove that in such a case the defining infimum is in fact the minimum. Moreover, there exist holomorphic curves $0 \neq \varphi:(\mathbb{C}, 0) \rightarrow\left(\mathbb{C}^{n}, 0\right)$ satisfying

$$
l_{0}(f)=\frac{\operatorname{ord} f \circ \varphi}{\operatorname{ord} \varphi},
$$

and such curves are optimal in the sense that for every $0 \neq \psi:(\mathbb{C}, 0) \rightarrow\left(\mathbb{C}^{n}, 0\right)$ it holds $l_{0}(f) \geqslant \frac{\operatorname{ord} f \circ \psi}{\text { ord } \psi}$. (For the proofs of these facts, see [LT08] or [Pło88].) It easily follows that, for the optimal curves $\varphi$,

$$
\|f \circ \varphi\| \sim\|\varphi\|^{l_{0}(f)},
$$

so in a way we recover the inequalities (1) that we started from.

*This document has been produced using $\mathrm{T}_{\mathrm{E}} \mathrm{X}_{\mathrm{MACS}}$ (see http://www.texmacs.org)

${ }^{\dagger}$ MSC 2010: 32S05, 58K05 Keywords: Łojasiewicz exponent, quasihomogeneous singularity 
Much is known about $l_{0}(f)$ for $n=2$ (see [LT08, KL77, Tei77, CK88, Pło88, CK95, Len98, Pło01]), the case $n \geqslant 3$ is however challenging still (for some recent results see e.g. [Biv09, KOP09, Ole09, Pło10, BKO12, BE12]).

In this paper we are interested in the Łojasiewicz exponent of a singularity $f:\left(\mathbb{C}^{n}, 0\right) \rightarrow(\mathbb{C}, 0)$,

$$
\mathscr{L}_{0}(f):=l_{0}\left(\frac{\partial f}{\partial z_{1}}, \ldots, \frac{\partial f}{\partial z_{n}}\right)
$$

More specifically, we give formulas for $\mathscr{L}_{0}(f)$ if $f$ is a (weakly) (semi-)quasihomogeneous singularity (see Definition 11), in terms of its weights. Such formulas are known when $n \leqslant 3$ ([KOP09] for the quasihomogeneous, and [BKO12] for the semiquasihomogeneous case). In [TYZ10], there appeared an incorrect proof of an analogous formula for general $n$ (cf. A MS review MR2679619 for the details). We aim at giving a valid proof of this result (Theorem 3).

Our approach to the problem follows closely that of [KOP09]: first we prove a general lemma which is interesting in its own right (Proposition 1), and then apply it to deal with the non-generic situations for the computation of the Łojasiewicz exponent. After proving Theorem 3 , we pass to the more general situation allowing „weak" weights. Here, most of the necessary ingredients are delivered by S A I T O ([Sai71]) whose results allow us to reduce the general problem to the semiquasihomogeneous one using stable equivalence (Corollary 3 and Theorem 4) and also express the value of the Łojasiewicz exponent of a quasihomogeneous singularity in a coordinate-independent fashion (Theorem 5). We conclude with the observation that Teissier's conjecture (see at the end of the paper) is valid in the class of weakly semiquasihomogeneous functions (Corollary 5).

The main results of the paper are Theorems 35 and Corollary 5 .

\section{Definitions and Known Facts}

The following definitions are much in the spirit of S A I T O [Sai71] and A R N OLD [Arn74].

Definition 1 Let $f:\left(\mathbb{C}^{n}, 0\right) \rightarrow(\mathbb{C}, 0)$ be a (germ of a) holomorphic function. Then:

$\rightarrow f$ is an (isolated) singularity, if it has an isolated critical point at 0

$\rightarrow f$ is weakly quasihomogeneous of type $\left(d ; l_{1}, \ldots, l_{n}\right)$, shortly: $f \in \mathrm{WQH}\left(d ; l_{1}, \ldots, l_{n}\right)$, if $l=\left(l_{1}, \ldots, l_{n}\right) \in$ $\mathbb{R}^{n}, d \in \mathbb{R}_{>0}$ and for every monomial $z^{a}=z_{1}^{a_{1}} \cdot \ldots \cdot z_{n}^{a_{n}}$ appearing in the expansion of $f$ with a non-zero coefficient it holds $\langle a, l\rangle:=a_{1} l_{1}+\ldots+a_{n} l_{n}=d$; in particular, $f=0$ is W Q $\mathrm{H}$ of all types

The numbers $l_{1}, \ldots, l_{n}$ will be called weights. The number $\operatorname{deg}_{l}\left(z^{a}\right):=\langle a, l\rangle$ will be referred to as the weighted degree of a monomial $z^{a}$. For a series $g(z)=\sum_{a \in \mathbb{N}_{0}^{n}} g_{a} z^{a}$, its weighted order is $\operatorname{ord}_{l} g:=\inf _{g_{a} \neq 0}\left(\operatorname{deg}_{l}\left(z^{a}\right)\right)$.

$\rightarrow f$ is quasihomogeneous of type $\left(d ; l_{1}, \ldots, l_{n}\right)$, shortly: $f \in \mathrm{QH}\left(d ; l_{1}, \ldots, l_{n}\right)$, if it is W Q H of type $\left(d ; l_{1}, \ldots\right.$, $\left.l_{n}\right)$ with $d, l_{1}, \ldots, l_{n} \in \mathbb{Q}$ and $l_{1} / d, \ldots, l_{n} / d \in(0,1 / 2]$

Note that from the definition it follows that a $\mathrm{QH} f$ is necessarily a (germ of a) polynomial of order greater than or equal to 2.

$\rightarrow f$ is weakly semiquasihomogeneous of type $\left(d ; l_{1}, \ldots, l_{n}\right)$, shortly: $f \in \mathrm{W} \mathrm{SQH}\left(d ; l_{1}, \ldots, l_{n}\right)$, if it can be written in the form $f=f_{0}+f^{\prime}$, where $f_{0}$ is a W Q H singularity of type $\left(d ; l_{1}, \ldots, l_{n}\right)$, ord $f^{\prime}>1$ and every monomial appearing in the expansion of $f^{\prime}$ is of weighted degree greater than $d$

The singularity $f_{0}$ will be called the principal part of $f$.

$\rightarrow f$ is semiquasihomogeneous of type $\left(d ; l_{1}, \ldots, l_{n}\right)$, shortly: $f \in \mathrm{SQH}\left(d ; l_{1}, \ldots, l_{n}\right)$, if it is W S Q H type $\left(d ; l_{1}, \ldots, l_{n}\right)$ with $d, l_{1}, \ldots, l_{n} \in \mathbb{Q}$ and $l_{1} / d, \ldots, l_{n} / d \in(0,1 / 2]$ (or $f_{0}$ is a $\mathrm{QH}$ singularity)

It is known that a S QH $f$ (or a W SQH $f$ with positive weights) is automatically an isolated singularity [Arn74].

Remark It is easy to see that the types $\left(d ; l_{1}, \ldots, l_{n}\right)$ in the definitions can always be normalized to $\left(1 ; l_{1} / d, \ldots\right.$, $\left.l_{n} / d\right)$. Saito, op. cit., allowed also complex weights for W QH functions, however, as he proved, it is often not restrictive to consider only the rational ones. On the other hand, Arnold, op. cit., considered mostly S Q H functions; the definition of W S QH functions is perhaps somewhat non-standard. 
The following theorem holds [KOP09, Thm. 1, Cor. 4 and Thm. 3].

Theorem 1 Let $f:\left(\mathbb{C}^{n}, 0\right) \rightarrow(\mathbb{C}, 0)$, where $n \leqslant 3$, be a weakly quasihomogeneous singularity of type $\left(1 ; l_{1}, \ldots\right.$, $\left.l_{n}\right)$ with positive rational weights. Put $w_{i}:=1 / l_{i}$. Then

$$
\mathscr{L}_{0}(f)=\min \left(\max _{1 \leqslant i \leqslant n}\left(w_{i}-1\right), \prod_{1 \leqslant i \leqslant n}\left(w_{i}-1\right)\right) .
$$

In particular, if $f$ is quasihomogeneous

$$
\mathscr{L}_{0}(f)=\max _{1 \leqslant i \leqslant n}\left(w_{i}-1\right)
$$

Remark Actually, formula (4) is proved in [KOP09] only for $n=3$. However, for a function $f$ of 2 indeterminates one can consider the function $f:=f+z_{3}^{2}$, which has the same Eojasiewicz exponent as $f$ and for which the weight $l_{3}=1 / 2$, and then apply formula 44 to it to find an analogous formula for $\mathscr{L}_{0}(f)$.

Theorem 1 is known to generalize to the case of a SQH function $f$ ([BKO12, Theorem 3.2]) in exactly the same form. Namely, taking account of the remark above, one can state:

Theorem 2 Let $f:\left(\mathbb{C}^{n}, 0\right) \rightarrow(\mathbb{C}, 0)$, where $n \leqslant 3$, be a weakly semiquasihomogeneous function with positive rational weights and principal part $f_{0}$. Then

$$
\mathscr{L}_{0}(f)=\mathscr{L}_{0}\left(f_{0}\right)
$$

\section{Results}

We begin with a proof of a proposition which is a weaker version of [KOP09, Thm. 2], but generalized to $n \geqslant 4$ indeterminates. We remark that in loc. cit. the theorem is stated as a very special case of local Hilbert's Nullstellensatz and the authors conjecture it to be true in any dimension (op. cit., Problem 1). It is however not the case, already for four indeterminates (cf. Example 1).

Notation For a germ $f \in \mathscr{O}^{n}$ of $n$ indeterminates and $i \in\{1, \ldots, n\}$ we define $\nabla f:=\left(\frac{\partial f}{\partial z_{1}}, \ldots, \frac{\partial f}{\partial z_{n}}\right)$ and ${ }^{\hat{i}} \nabla f:=$ $\left(\frac{\partial f}{\partial z_{1}}, \ldots, \widehat{\frac{\partial f}{\partial z_{i}}}, \ldots, \frac{\partial f}{\partial z_{n}}\right)$, where the hat means omission. For a set $F$ of germs, $\mathscr{V}(F)$ will denote the germ at 0 of the set of common zeroes of the system $F$.

Proposition 1 Let $f:\left(\mathbb{C}^{n}, 0\right) \rightarrow(\mathbb{C}, 0)$ be an isolated singularity such that $\mathscr{V}\left({ }^{1} \nabla f\right) \subset \mathscr{V}\left(z_{1}\right)$. Then ord $f=2$. Moreover, there exists an $1<i \leqslant n$ such that the monomial $z_{1} z_{i}$ does appear in the expansion of $f$ with a non-zero coefficient while the monomial $z_{i}^{2}$ does not.

Proof Let us consider the deformation $f_{s}(z):=f(z)+s z_{1}^{2}$ of the germ $f$. For any $s \in \mathbb{C}$, it is $\mathscr{V}\left({ }^{1} \nabla f_{s}\right)=$ $\mathscr{V}\left({ }^{\hat{l}} \nabla f\right)=: \mathscr{A}$ because in fact $\left({ }^{\hat{1}} \nabla f_{s}\right) \mathbb{C}\left\{z_{1}, \ldots, z_{n}\right\}=\left({ }^{\hat{1}} \nabla f\right) \mathbb{C}\left\{z_{1}, \ldots, z_{n}\right\}$ as ideals. Take a set $\Phi$ of non-equivalent parametrizations of the curve $\mathscr{A}$. Then one has

$$
\mu\left(f_{s}\right)=\sum_{\varphi \in \Phi} l_{\varphi} \text { ord }\left(\frac{\partial f_{s}}{\partial z_{1}} \circ \varphi\right)
$$

where the numbers $l_{\varphi}$ are the multiplicities of the branches $\varphi$ of the curve $\mathscr{A}$. But by assumption, $\varphi \subset \mathscr{V}\left({ }^{1} \nabla f\right) \subset$ $\mathscr{V}\left(z_{1}\right)$ and hence ord $\left(\frac{\partial f_{s}}{\partial z_{1}} \circ \varphi\right)=\operatorname{ord}\left(\frac{\partial f}{\partial z_{1}} \circ \varphi\right)$, for every $\varphi \in \Phi$. Thus, one has $\mu\left(f_{s}\right)=\mu(f), s \in \mathbb{C}$, or in another words $-\left(f_{s}\right)$ is a $\mu$-constant deformation of $f$. Using a result of TROT M A (see [Tro80] or [PT12, Prop. 1.1]), we conclude that the family $\left(f_{s}\right)$ is equimultiple. Since $f$ is a singularity, ord $f_{s}=2$ for $s$ close to 0 , and hence also ord $f=2$. 
Let $q(z)$ be the quadratic form of $f$. Assume, to the contrary, that the form does not depend on $z_{1}$. Then, by the splitting lemma, $f$ can be transformed through a biholomorphic change of coordinates $\Psi$ into $\tilde{f}=\tilde{h}\left(z_{1}, \ldots, z_{k}\right)+$ $z_{k+1}^{2}+\ldots+z_{n}^{2}$, where ord $\tilde{h} \geqslant 3$ and $k \geqslant 1$. Moreover, it is easy to see that $\Psi$ can be chosen so that $\Psi(z)=\left(z_{1}, \ldots\right)$ (by assumption; just recall that $\Psi$ engages essentially at most those variables that appear in the form $q(z)$ ). However, such change of parameters does not drag the zero set of ${ }^{\hat{1}} \nabla f$ out of the hyperplane $z_{1}=0$, i.e. $\mathscr{V}\left({ }^{1} \nabla \tilde{f}\right) \subset \mathscr{V}\left(z_{1}\right)$. Thus also $\mathscr{V}\left({ }^{1} \nabla \tilde{h}\right) \subset \mathscr{V}\left(z_{1}\right)$, which is impossible by what we already know. It follows that $q$ does depend on $z_{1}$. More precisely, since every $f-\vartheta z_{1}^{2}, \vartheta \in \mathbb{C}$, also fulfills the conditions of the proposition, we deduce that in the expansion of $f$ there has to appear a monomial of the form $z_{1} z_{i}, i \neq 1$. If for every such $i$, in $q(z)$ there appeared also the monomial $z_{i}^{2}$, then we would be able to transform the function $f$ into one that does not contain $z_{1}$ in its quadratic form, possibly except for $z_{1}^{2}$, but still one that fulfills the assumptions of the proposition; contradiction.

Remark For $f$ (semi)quasihomogeneous, instead of Trotman's theorem one can use the results of [Gre86] or O'S87 to prove that ord $f=2$.

Example 1 Although we will not prove it, we remark that using Proposition 1 it is possible to show that every singularity $f:\left(\mathbb{C}^{n}, 0\right) \rightarrow(\mathbb{C}, 0)$ satisfying $\mathscr{V}\left({ }^{1} \nabla f\right) \subset \mathscr{V}\left(z_{1}\right)$ can be transformed into the form $f=z_{1} z_{2}+$ $g\left(z_{2}, \ldots, z_{n}\right)+h\left(z_{1}\right)$ by a formal change of coordinates whose first component is the identity. Thus, in order to prove that under the assumptions of Proposition 1 it holds $z_{1} \in\left({ }^{1} \nabla f\right)$ (cf. [KOP09. Problem 1]), it is enough to check this for singularities of the form indicated above. Now, for $n=3$ it turns out that $g$ can be further transformed into one that does not depend on $z_{2}$, which gives an alternative proof of op. cit. Theorem 2 . For $n \geqslant 4$, let us consider any singularity $g_{0}=g_{0}\left(z_{3}, \ldots, z_{n}\right)$ such that $g_{0} \notin\left(\frac{\partial g_{0}}{\partial z_{3}}, \ldots, \frac{\partial g_{0}}{\partial z_{n}}\right)$, i.e. a $g_{0}$ that is not quasihomogeneous in any system of coordinates, and put $f:=z_{1} z_{2}+\left(1+z_{2}\right) g_{0}$. Assume, to the contrary, that $z_{1} \in\left({ }^{1} \nabla f\right) \mathbb{C}\left\{z_{1}, \ldots, z_{n}\right\}$. It is easy to see that there has to exist a relation of the form $z_{1}=\frac{\partial f}{\partial z_{2}}+A_{3} \frac{\partial f}{\partial z_{3}}+\ldots+A_{n} \frac{\partial f}{\partial z_{n}}$, where $A_{j} \in \mathbb{C}\left\{z_{2}, \ldots, z_{n}\right\}$. But this relation implies that $g_{0} \in\left(\frac{\partial f}{\partial z_{3}}, \ldots, \frac{\partial f}{\partial z_{n}}\right) \mathbb{C}\left\{z_{2}, \ldots, z_{n}\right\}$ and hence - that also $g_{0} \in\left(\frac{\partial g_{0}}{\partial z_{3}}, \ldots, \frac{\partial g_{0}}{\partial z_{n}}\right) \mathbb{C}\left\{z_{3}, \ldots, z_{n}\right\}$, contradiction. As a more specific example, one can consider for instance $f:=z_{1} z_{2}+\left(1+z_{2}\right)\left(z_{3}^{4}+z_{3}^{2} z_{4}^{3}+z_{4}^{5}\right)$. It can be checked, using a computer algebra system, that $z_{1} \notin(\nabla f)$ but $z_{1}^{2} \in\left({ }^{1} \nabla f\right)$.

We suspect that it may be the case that $\mathscr{V}\left({ }^{1} \nabla f\right) \subset \mathscr{V}\left(z_{1}\right)$ for a singularity $f$ implies $z_{1}^{n-2} \in\left({ }^{1} \nabla f\right)$, for $n \geqslant 3$.

Using Proposition 1 we easily deduce the following.

Corollary 1 For every semiquasihomogeneous function $f$ of type $\left(d ; l_{1}, \ldots, l_{n}\right)$ such that $0<l_{j} / d<1 / 2$, $j=1, \ldots, n$, and every $i \in\{1, \ldots, n\}$ it is

$$
\mathscr{V}\left({ }^{\hat{i}} \nabla f\right) \not \subset \mathscr{V}\left(z_{i}\right) .
$$

Proof Since then $f$ is of order greater than 2 .

Corollary 2 Let $f:\left(\mathbb{C}^{n}, 0\right) \rightarrow(\mathbb{C}, 0)$ be a quasihomogeneous singularity of type $\left(1 ; l_{1}, \ldots, l_{n}\right)$. Assume that $l_{1} \leqslant \ldots \leqslant l_{n}$ and $\mathscr{V}\left({ }^{1} \nabla f\right) \subset \mathscr{V}\left(z_{1}\right)$. Then $f$ is a homogeneous polynomial of order 2 . In particular, $\mathscr{L}_{0}(f)=1$.

Proof By Proposition 1 in $f$ there appears a monomial $z_{1} z_{i}$ with a non-zero coefficient. It follows that $l_{1}=l_{i}=1 / 2$ and hence also all the other weights are equal to $1 / 2$. Now we can apply [Pło85, Lemme 2.4] to conclude that $\mathscr{L}_{0}(f)=\max _{1 \leqslant j \leqslant n}\left(\operatorname{ord} \frac{\partial f}{\partial z_{j}}\right)=1$.

Theorem 3 Let $f:\left(\mathbb{C}^{n}, 0\right) \rightarrow(\mathbb{C}, 0)$ be a semiquasihomogeneous function of type $\left(1 ; l_{1}, \ldots, l_{n}\right)$. Put $w_{i}:=1 / l_{i}$. Then

$$
\mathscr{L}_{0}(f)=\max _{1 \leqslant i \leqslant n}\left(w_{i}-1\right)
$$

Proof First assume that $f$ is quasihomogeneous. Let $l_{1} \leqslant \ldots \leqslant l_{n}$. If $\mathscr{V}\left({ }^{\hat{\imath}} \nabla f\right) \subset \mathscr{V}\left(z_{1}\right)$ then formula $(5)$ is valid by Corollary 2 . In the opposite case, it its enough to apply [KOP09, Proposition 2]. 
For $f$ semiquasihomogeneous, Corollary 4.8 of [BE12] or Proposition 4.1 of [BKO12] assert that $\mathscr{L}_{0}\left(f_{0}\right) \leqslant$ $\mathscr{L}_{0}(f)$, where $f_{0}$ is the principal part of $f$. In another words, $\max _{1 \leqslant i \leqslant n}\left(w_{i}-1\right) \leqslant \mathscr{L}_{0}(f)$. By [Pło85, Proposition $2.2]$ we obtain the opposite inequality.

It remains to consider the case of ,,weak weights”. For this purpose, we adopt the results of [Sai71] to W S QH functions. First, we prove a WSQH variant of SPLITTING LEMMA.

Lemma 1 Let $f:\left(\mathbb{C}^{n}, 0\right) \rightarrow(\mathbb{C}, 0)$ be a weakly semiquasihomogeneous function of type $(1 ; l):=(1 ; \underbrace{p_{1}, \ldots, p_{i}}_{x}$, $\underbrace{q_{1}, \ldots, q_{k}}_{y}, \underbrace{r_{1}, \ldots, r_{i}}_{z})$, where $2 i+k=n$, and of the form

$$
f(x, y, z)=\left(f_{0}(y)+\sum_{j=1}^{i} x_{j} z_{j}\right)+f^{\prime}(x, y, z)
$$

where $(x, y, z):=\left(x_{1}, \ldots, x_{i}, y_{1}, \ldots, y_{k}, z_{1}, \ldots, z_{i}\right)$ and $f_{0}(y)+\sum_{j=1}^{i} x_{j} z_{j}$ is the principal part of $f$. Assume that $q_{1}, \ldots, q_{k}>0$. Then either $f$ is stably equivalent to a weakly semiquasihomogeneous singularity of type $\left(1 ; q_{1}, \ldots\right.$, $q_{k}$ ) having $f_{0}(y)$ as its principal part (if $k>0$ ) or is of type $\mathscr{A}_{1}$ (if $k=0$ ).

Proof For $k=0$ it is enough to apply the ordinary splitting lemma; hence in the following we will assume that $k>0$. Also we exclude the case $i=0$, as it is trivial.

First note that by our assumptions it is $p_{j}+r_{j}=1(j=1, \ldots, i)$. Let $\mathrm{M}:=\mu\left(f_{0}\right)<\infty$ and let $g \in \mathrm{W} \mathrm{SQH}(1 ; l)$ be such that $\operatorname{ord}_{l}(f-g)>\mathrm{M}$ and of the form $g(x, y, z)=\left(f_{0}(y)+\sum_{j=1}^{i} x_{j} z_{j}\right)+g^{\prime}(x, y, z),\left(f_{0}(y)+\sum_{j=1}^{i} x_{j} z_{j}\right)$ being the principal part of $g$ and $g^{\prime}$ being a non-zero polynomial. Recall that by Definition 1 necessarily ord $g>1$. We claim that for each $m \in \mathbb{N}$ it is possible to write $g$ as

$$
g(x, y, z)=f_{0}(y)+\mathrm{H}^{(m)}(y)+\Lambda^{(m)}(x, y, z)
$$

where $\operatorname{ord}_{l} \mathrm{H}^{(m)}>1$,

$$
\Lambda^{(m)}(x, y, z)=\sum_{1 \leqslant j \leqslant i}\left(x_{j}+\Gamma_{j}^{(m)}(x, y, z)\right)\left(z_{j}+\Delta_{j}^{(m)}(x, y, z)\right)+R^{(m)}(x, y, z),
$$

$\operatorname{ord}_{l} \Gamma_{j}^{(m)} \geqslant d^{(1)}-r_{j}, \operatorname{ord}_{l} \Delta_{j}^{(m)} \geqslant d^{(1)}-p_{j}(j=1, \ldots, i)$ and $\operatorname{ord}_{l} R^{(m)} \geqslant m\left(d^{(1)}-1\right)+1$ with $d^{(1)}:=\operatorname{ord}_{l} g^{\prime} \in$ $(1, \infty)$. Moreover $\mathrm{H}^{(m)}, \Lambda^{(m)}, \Gamma_{j}^{(m)}, \Delta_{j}^{(m)}, R^{(m)}$ are polynomials in $x, y, z$, vanishing at 0 .

Indeed, for $m=1$ it is enough to put $R^{(1)}:=g^{\prime}(x, y, z)$ and $\Lambda^{(1)}:=\sum_{j=1}^{i} x_{j} z_{j}+R^{(1)}$ so that $\Gamma_{j}^{(1)}:=\Delta_{j}^{(1)}:=0$ $(j=1, \ldots, i)$ and $\mathrm{H}^{(1)}:=0$.

Now, assuming $\left(7\right.$ and 8 for some $m \in \mathbb{N}$, we decompose $R^{(m)}$ into polynomials in the following way:

$$
R^{(m)}=\eta^{(m+1)}(y)+\sum_{1 \leqslant j \leqslant i}\left(x_{j} \delta_{j}^{(m+1)}(x, y, z)+z_{j} \gamma_{j}^{(m+1)}(x, y, z)\right),
$$

where $\operatorname{ord}_{l} \eta^{(m+1)}>1$ and $x_{j} \delta_{j}^{(m+1)}, z_{j} \gamma_{j}^{(m+1)}$ are of weighted order greater than or equal to $\operatorname{ord}_{l} R^{(m)}$. We put $\mathrm{H}^{(m+1)}:=\mathrm{H}^{(m)}+\eta^{(m+1)}, \Lambda^{(m+1)}:=\Lambda^{(m)}-\eta^{(m+1)}(y), \Gamma_{j}^{(m+1)}:=\Gamma_{j}^{(m)}+\gamma_{j}^{(m+1)}$ and $\Delta_{j}^{(m+1)}:=\Delta_{j}^{(m)}+\delta_{j}^{(m+1)}$ $(j=1, \ldots, i)$; clearly, these are polynomials, vanishing at 0 . Moreover, $\operatorname{ord}_{l} \mathrm{H}^{(m+1)}>1$,

$$
\begin{aligned}
& \operatorname{ord}_{l} \gamma_{j}^{(m+1)} \geqslant \operatorname{ord}_{l} R^{(m)}-\operatorname{ord}_{l} z_{j} \geqslant m\left(d^{(1)}-1\right)+1-r_{j} \geqslant d^{(1)}-r_{j}, \\
& \operatorname{ord}_{l} \delta_{j}^{(m+1)} \geqslant \operatorname{ord}_{l} R^{(m)}-\operatorname{ord}_{l} x_{j} \geqslant m\left(d^{(1)}-1\right)+1-p_{j} \geqslant d^{(1)}-p_{j}
\end{aligned}
$$

and hence $\operatorname{ord}_{l} \Gamma_{j}^{(m+1)} \geqslant d^{(1)}-r_{j}, \operatorname{ord}_{l} \Delta_{j}^{(m+1)} \geqslant d^{(1)}-p_{j}(j=1, \ldots, i)$. By \&8 and 90 we have

$$
\begin{aligned}
\Lambda^{(m+1)} & =\sum_{1 \leqslant j \leqslant i}\left(x_{j}+\Gamma_{j}^{(m)}\right)\left(z_{j}+\Delta_{j}^{(m)}\right)+R^{(m)}-\eta^{(m+1)}= \\
& =\sum_{1 \leqslant j \leqslant i}\left(x_{j}+\Gamma_{j}^{(m)}\right)\left(z_{j}+\Delta_{j}^{(m)}\right)+\sum_{1 \leqslant j \leqslant i}\left(x_{j} \delta_{j}^{(m+1)}+z_{j} \gamma_{j}^{(m+1)}\right)= \\
& =\sum_{1 \leqslant j \leqslant i}\left(x_{j}+\Gamma_{j}^{(m+1)}\right)\left(z_{j}+\Delta_{j}^{(m+1)}\right)+R^{(m+1)},
\end{aligned}
$$


where $R^{(m+1)}:=-\sum_{1 \leqslant j \leqslant i}\left(\Gamma_{j}^{(m)} \delta_{j}^{(m+1)}+\gamma_{j}^{(m+1)} \Delta_{j}^{(m+1)}\right)$. Using 11 and induction hypothesis, ord $\Gamma_{j}^{(m)} \delta_{j}^{(m+1)}=$ $\operatorname{ord}_{l} \Gamma_{j}^{(m)}+\operatorname{ord}_{l} \delta_{j}^{(m+1)} \geqslant\left(d^{(1)}-r_{j}\right)+\left(m\left(d^{(1)}-1\right)+1-p_{j}\right)=(m+1)\left(d^{(1)}-1\right)+1(j=1, \ldots, i)$ and similarly for the other terms. Hence also $\operatorname{ord}_{l} R^{(m+1)} \geqslant(m+1)\left(d^{(1)}-1\right)+1$. Thus, (7) and 8 hold for $m+1$ and - by induction - for all $m \in \mathbb{N}$.

Fix any $m \in \mathbb{N}$ and consider $\tilde{g}_{m}:=g-R^{(m)}$. Since $\operatorname{ord}_{l} \Gamma_{j}^{(m)} \geqslant d^{(1)}-r_{j}>1-r_{j}=p_{j}=\operatorname{deg}_{l} x_{j}$ and similarly $\operatorname{ord}_{l} \Delta_{j}^{(m)} \geqslant d^{(1)}-p_{j}>\operatorname{deg}_{l} z_{j}$ for $j=1, \ldots, i$, putting $\Psi(x, y, z):=\left(x_{1}+\Gamma_{1}^{(m)}, \ldots, x_{i}+\Gamma_{i}^{(m)}, y, z_{1}+\Delta_{1}^{(m)}, \ldots, z_{i}+\right.$ $\left.\Delta_{i}^{(m)}\right)$ we easily see that $\Psi(0)=0$ and the matrix $\left.\frac{\partial \Psi}{\partial(x, y, z)}\right|_{(x, y, z)=0}$ is, up to permutation of rows, a triangular one. Composing $\tilde{g}_{m}$ with $\Psi^{-1}$ and then with a linear transformation, we find by $(7)$ and $(8)$ that $\tilde{g}_{m}$ goes into the form

$$
\breve{g}_{m}(x, y, z):=f_{0}(y)+\mathrm{H}^{(m)}(y)+\sum_{j=1}^{i}\left(x_{j}^{2}+z_{j}^{2}\right) .
$$

Since (by our assumptions and Definition 1) $f_{0}$ is a W Q H singularity of type $(1 ; q):=\left(1 ; q_{1}, \ldots, q_{k}\right)$ and $_{\text {ord }} \mathrm{H}^{(m)}$ $=\operatorname{ord}_{l} \mathrm{H}^{(m)}>1$, we deduce that $\breve{g}_{m}$ is W S Q H of type $\left(1 ; \frac{1}{2}, \ldots, \frac{1}{2}, q_{1}, \ldots, q_{k}, \frac{1}{2}, \ldots, \frac{1}{2}\right)$, and since all these weights are positive, we conclude that $\breve{g}_{m}$ is a singularity. More precisely, $\mu\left(\breve{g}_{m}\right)=\mu\left(f_{0}\right)=M<\infty$ (by the very same reasoning as in [Arn74, Theorem 3.1] and by the stable-equivalence-invariancy of the Milnor number). Hence, the degree of (right) determinacy of $\breve{g}_{m}$ - and thus also of $\tilde{g}_{m}-$ can be bounded from above by $M$.

Now recall that $\operatorname{ord}_{l} R^{(m)} \geqslant m\left(d^{(1)}-1\right)+1 \underset{m \rightarrow \infty}{\longrightarrow} \infty$, because $d^{(1)}>1$. Since there are only finitely many monomials of given (ordinary) degree, it follows that $\overline{\lim }_{m \rightarrow \infty}$ ord $R^{(m)}=\infty$. Hence, there exists an $m_{0} \in \mathbb{N}$ for which the order of $R^{\left(m_{0}\right)}$ is higher than the number $M . \tilde{g}_{m_{0}}$ being $M$-determined, this means that $\tilde{g}_{m_{0}}$ is biholomorphically equivalent to $g=\tilde{g}_{m_{0}}+R^{\left(m_{0}\right)}$ and thus we conclude that also $g$ is $M$-determined and biholomorphically equivalent to $\breve{g}_{m_{0}}$. But in such a case $f$ is right equivalent to $g$ and hence - to $\breve{g}_{m_{0}}$. Finally, from (12) it follows that $f$ is stably equivalent to the W S Q H singularity $f_{0}(y)+\mathrm{H}^{\left(m_{0}\right)}(y)$ of type $(1 ; q)$ and of principal part equal to $f_{0}$. The lemma is proved.

Corollary 3 Let $f:\left(\mathbb{C}^{n}, 0\right) \rightarrow(\mathbb{C}, 0)$ be a weakly semiquasihomogeneous function of type $\left(1 ; l_{1}, \ldots, l_{n}\right)$. Let $l_{1} \leqslant \ldots \leqslant l_{i} \leqslant 0<l_{i+1} \leqslant \ldots \leqslant l_{i+k}<1 \leqslant l_{i+k+1} \leqslant \ldots \leqslant l_{n}$. Then $f$ is a singularity and is either stably equivalent to a weakly semiquasihomogeneous function of type $\left(1 ; l_{i+1}, \ldots, l_{i+k}\right)$ if $k \neq 0$, or is of type $\mathscr{A}_{1}$ if $k=0$.

Proof By [Sai71, Korollar 1.9], $i=n-i-k$ and moreover $l_{j}+l_{n+1-j}=1$, for $1 \leqslant j \leqslant i$. Repeating the proof of [Sai71, Lemma 1.10] one concludes that the principal part $f_{0}$ of $f$ can be written in the following form

$$
f_{0}(x, y, z)=\tilde{f}_{0}(y)+\sum_{j=1}^{i}\left(g_{j}(z)+h_{j}(x, y, z)\right) x_{i+1-j}
$$

where the coordinates in $\left(\mathbb{C}^{n}, 0\right)$ are denoted by $(x, y, z):=\left(x_{1}, \ldots, x_{i}, y_{i+1}, \ldots, y_{i+k}, z_{i+k+1}, \ldots, z_{n}\right)$, the map-germ $\left(g_{1}, \ldots, g_{i}\right)$ is a biholomorphism of $\left(\mathbb{C}^{i}, 0\right)$ and the functions $h_{j}$ satisfy $h_{j}(0,0, z)=0$. It follows that $G(x, y, z):=$ $\left(x, y, g_{1}(z)+h_{1}(x, y, z), \ldots, g_{i}(y)+h_{i}(x, y, z)\right)$ is a biholomorphism of $\left(\mathbb{C}^{n}, 0\right)$. Moreover, 13$)$ implies that each component $G_{j}$ of $G$ is $\mathrm{WQH}$ of type $\left(l_{j} ; l_{1}, \ldots, l_{n}\right)$, for $j=1, \ldots, n$. Using the identity $\mathrm{Id}=G^{-1} \circ G$ we easily check that each component $G_{j}^{-1}$ of $G^{-1}$ is also $\mathrm{WQH}$ of type $\left(l_{j}, l_{1}, \ldots, l_{n}\right)$, for $j=1, \ldots, n$. Hence, for every monomial $w$ the function $\left(G^{-1}\right)^{*} w$ is W QH of type $\left(\operatorname{deg}_{l} w ; l_{1}, \ldots, l_{n}\right)$. It follows that $\tilde{f}:=\left(G^{-1}\right)^{*} f$ is W S Q of type $\left(1 ; l_{1}, \ldots, l_{n}\right)$. Writing $f=f_{0}+f^{\prime}$, we have

$$
\tilde{f}(x, y, z)=\left(G^{-1}\right)^{*} f(x, y, z)=\left(\tilde{f}_{0}(y)+\sum_{j=1}^{i} z_{i+k+j} x_{i+1-j}\right)+\left(G^{-1}\right)^{*} f^{\prime}(x, y, z) .
$$

Since the weights $l_{i+1}, \ldots, l_{i+k}$ are positive, we can apply Lemma 1 to $\tilde{f}$. Clearly, this gives the required assertions also for $f=G^{*} \tilde{f}$. 
Corollary 4 Let $f:\left(\mathbb{C}^{3}, 0\right) \rightarrow(\mathbb{C}, 0)$ be a weakly semiquasihomogeneous function of type $\left(1 ; l_{1}, l_{2}, l_{3}\right)$ with
rational weights. Put $w_{i}:=\left\{\begin{array}{ll}1 / l_{i}, & \text { if } l_{i} \notin\{0,1\} \\ 2, & \text { if } l_{i} \in\{0,1\}\end{array}\right.$. Then formula 44 holds with $n=3$.

Proof Let $l_{1} \leqslant l_{2} \leqslant l_{3}$. Assume that $l_{1} \leqslant 0$. Again by [Sai71, Korollar 1.9] we conclude that $l_{3}=1-l_{1}$ and $0<l_{2}<1$. Corollary 3 asserts that $f$ is stably equivalent to a W S Q H function $\tilde{f}$ of type $\left(1 ; l_{2}\right)$. Hence, $0<l_{2} \leqslant \frac{1}{2}$ and $\mathscr{L}_{0}(\tilde{f})=$ ord $\tilde{f}-1=\frac{1}{l_{2}}-1$. Since the Łojasiewicz exponent is an invariant of the stable equivalence, $\mathscr{L}_{0}(f)=$ $\frac{1}{l}-1$. We easily check that (4) has the same value. The case of all weights being positive is covered by Theorems 11 and 2

We remark that the above corollary is not true for $n=2$ unless one modifies formula (4) slightly. Similarly, it has been shown by M. Sękalski [Sęk10] that formula (4) of Theorem 1 fails in dimensions $n \geqslant 4$, even for W QH singularities with positive rational weights. A version of formula (4), valid in any dimension, is given below, in Theorem 4

We illustrate Corollary 4 with the following example.

Example 2 Let us consider $f(x, y, z)=x y+x^{4} y^{3}+(z+y)^{3}$. The singularity $f$ is W S Q H of type $(1 ;-2,3,1 / 3)$ with principal part $f_{0}=x y+x^{4} y^{3}+z^{3}$ and also it is W SQH of type $(1 ; 2 / 3,1 / 3,1 / 3)$ with principal part $\tilde{f}_{0}=$ $x y+(z+y)^{3}$. Corollary 4 asserts that the Łojasiewicz exponent of $f$ is equal to 2 , in both cases.

Theorem 4 Let $f:\left(\mathbb{C}^{n}, 0\right) \rightarrow(\mathbb{C}, 0)$ be a weakly semiquasihomogeneous function of type $\left(1 ; l_{1}, \ldots, l_{n}\right)$. Define the multisets $L:=\left[l_{1}, \ldots, l_{n}\right], L^{-}:=\left[1-a: a \in L \wedge a>\frac{1}{2}\right]$ and the set $L^{0}:=\left(L \backslash L^{-}\right) \cup\left\{\frac{1}{2}\right\}$. Put $l_{\min }:=\min L^{0}$. Then $\mathscr{L}_{0}(f)=\frac{1}{l_{\min }}-1$.

Proof By [Sai71, Korollar 1.9] the number of the weights $\leqslant 0$ is the same as the number of the weights $\geqslant 1$ and such weights are distributed symmetrically with respect to $1 / 2$, counting multiplicities. Hence, upon applying Corollary 3 , we can assume that $0<l_{j}<1, j=1, \ldots, n$. (This affects neither $\mathscr{L}_{0}(f)$ nor the number $l_{\min }$, also in the degenerate case of all the weights lying outside the interval $(0,1)$.)

Now we repeat the last part of the proof of [Sai71, Satz 1.3]. Let $0<l_{1} \leqslant \ldots \leqslant l_{n}<1$ and assume that $l_{n}>\frac{1}{2}$. This means that the principal part $f_{0}(z)$ of $f(z)$ can depend only linearly on $z_{n}$. However, $f_{0}$ is a singularity and hence its expansion has to involve a monomial of the form $z_{n} z_{i}$, for some $i<n$ (cf. [Sai71, Korollar 1.6]). It is easy to see that $f_{0}$ can be brought to the form $\tilde{f}_{0}\left(z_{1}, \ldots, \hat{z}_{i}, \ldots, \hat{z}_{n}\right)+z_{i} z_{n}$ by a biholomorphism that does not violate the WSQH type of $f$. Hence, by this biholomorphism, $f$ is transformed to a W SQH function $\tilde{f}$ with principal part of the form $\tilde{f}_{0}\left(z_{1}, \ldots, \hat{z}_{i}, \ldots, \hat{z}_{n}\right)+z_{i} z_{n}$, which is the one assumed in $(6)$. Applying Lemma 1 to $\tilde{f}$ we can reduce it to $\left(\tilde{f}_{0}+\breve{f}^{\prime}\right)\left(z_{1}, \ldots, \hat{z}_{i}, \ldots, \hat{z}_{n}\right)+z_{i}^{2}+z_{n}^{2}$, where $\tilde{f}_{0}+\breve{f}^{\prime}$ is a W S Q H function of type $\left(1 ; l_{1}, \ldots, \hat{l}_{i}, \ldots, l_{n-1}, \hat{l}_{n}\right)$ or the zero function for $n=2$, in which case $\mathscr{L}_{0}(f)=1=\frac{1}{l_{\min }}-1$. In the former case, we easily see that the replacement of $f$ with $\tilde{f}_{0}+\breve{f}^{\prime}$ again does not affect the numbers $\mathscr{L}_{0}(f)$ and $l_{\min }$. Thus we reduce the number of variables. Continuing in this way, either we end up with a sum of squares at some stage, in which case $\mathscr{L}_{0}(f)=1=\frac{1}{l_{\min }}-1$, or with a W SQH function $\bar{f}$ with weights lying in the interval $\left(0, \frac{1}{2}\right]$. It is easy to see that such weights have to be rational, so in this last case $\bar{f}$ is in fact a SQH function. Moreover, for such weights the multiset $L^{-}$is empty and $l_{\min }$ is just the minimal of the weights. By formula (5), we get the desired equality.

Commentary If $f_{0}$ is the principal part of a W Q $\mathrm{H}$ function $f$, Theorem 4 implies that $\mathscr{L}_{0}(f)=\mathscr{L}_{0}\left(f_{0}\right)<\infty$. This once again signifies that a W S QH function $f$ is automatically an isolated singularity, which - by the above proof and Corollary 3 - is stably (and even biholomorphically) equivalent to a SQH function. Similarly, one can show that $\mu_{0}(f)=\mu_{0}\left(f_{0}\right)$.

For a W QH singularity $f$ one can compute its Łojasiewicz exponent in any system of coordinates. Namely, we have:

Theorem 5 Let $f:\left(\mathbb{C}^{n}, 0\right) \rightarrow(\mathbb{C}, 0)$ be an isolated singularity such that

$$
f=g_{1} \frac{\partial f}{\partial z_{1}}+\ldots+g_{n} \frac{\partial f}{\partial z_{n}}
$$




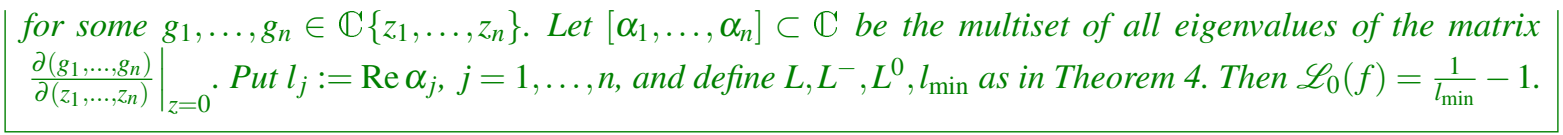

Proof Since $f$ is a singularity, [Sai71, Lemma 4.2] implies that $g_{1}(0)=\ldots=g_{n}(0)=0$. Next, [Sai71, Korollar 3.3] asserts that there exists a formal system of coordinates in which $f$ is a W QH formal singularity $\tilde{f}$ of type $\left(1 ; \alpha_{1}, \ldots, \alpha_{n}\right)$. (Here we allow the weights to be complex numbers and $\tilde{f}$ to be a formal power series.) But then, in this system of coordinates, $\tilde{f}$ is also W QH of type $\left(1 ; l_{1}, \ldots, l_{n}\right)$. Since $\tilde{f}$ is finitely determined, we can assume it is a polynomial and by ARTIN's Theorem [Art68 - that it is actually biholomorphically equivalent to $f$. The theorem follows upon applying Theorem 4

Remark Using (2) one can define the Łojasiewicz exponent also for $f \in \mathbb{C}\left[\left[z_{1}, \ldots, z_{n}\right]\right]$. With this definition, all the above results on $\mathscr{L}_{0}$ are true in the formal setting.

Example 3 Let $f:=x z+x y z^{2}+x y^{3}+y^{3} z^{2}+y^{5}+y^{2} z^{4}+z^{8}$. Using a computer algebra system one can check that $f \in(\nabla f)$ and (non-unique) eigenvalues of the Jacobian matrix of $\left(g_{1}, g_{2}, g_{3}\right)$ at 0 are $[-291960341316169405$ $4973386275881695714936.56 \ldots, 1 / 5,2919603413161694054973386275881695714937.56 \ldots]$. By Theorem 5 $\mathscr{L}_{0}(f)=4$. We also remark that in this case the value of $\mathscr{L}_{0}(f)$ can be computed using either Theorem 2 (because $\left.f \in \operatorname{SQH}\left(1 ; \frac{1}{2}, \frac{1}{5}, \frac{1}{2}\right)\right)$ or [Ole13, Thm. $1.8\left(1^{\circ}\right)$ ], because $f$ is Kouchnirenko non-degenerate and its Newton diagram consists of exceptional faces only (see op. cit. for the details).

We end the paper with a corollary concerning the conjecture of TEISSIER. Namely, just as there is the famous Z A R IS K I problem on multiplicity, the same question can be asked for the Łojasiewicz exponent: is it a topological invariant of a singularity? Since the question seems to be very difficult (although it is known to have the affirmative answer in case of germs of two indeterminates, see [Tei77] or [Pło01], and also for Q H singularities of three indeterminates [KOP09, Corollary 2]), it is natural to ask a weaker one:

Question (Teissier's conjecture) If $\left(f_{s}\right)$ is a topologically trivial deformation of a singularity $f_{0}$, does $\mathscr{L}_{0}\left(f_{0}\right)=$ $\mathscr{L}_{0}\left(f_{s}\right)$, for small $s \in \mathbb{C}$ ?

It should be noted that it is already known that Łojasiewicz exponent is lower semi-continuous in $\mu$-constant families ([Tei77], [Pło10]).

For S QH (and also W S QH) singularities we can answer Teissier's question in the affirmative.

Corollary 5 If $f:\left(\mathbb{C}^{n}, 0\right) \rightarrow(\mathbb{C}, 0)$ is a weakly semiquasihomogeneous function then the Eojasiewicz exponent $\mathscr{L}_{0}$ is constant on every topologically trivial deformation of $f$.

Proof Assume first that $f$ is SQH. From Theorem 3 it follows that the Łojasiewicz exponent of $f$ is determined by its weights. On the other hand, the Theorem of VARCHENKO [Var82] implies that the weights are invariant in $\mu$-constant deformations of $f$.

If we assume only that $f$ is W S Q H, then we may biholomorphically transform $f$ into a SQH function $\tilde{f}$ (cf. the commentary after Theorem 44. This transformation also carries any deformation $f_{s}$ of $f$ to a deformation $\tilde{f}_{s}$ of $\tilde{f}$; and the Milnor and Łojasiewicz numbers remain unchanged. Thus, we reduce the problem to the first case.

\section{References}

[Arn74] Vladimir Igorevich Arnold. Normal forms of functions in neighborhoods of degenerate critical points. Russ. Math. Surv., 29(2):10-50, 1974. Collection of articles dedicated to the memory of Ivan Georgievič Petrovskiĭ (1901-1973), I.

[Art68] Michael Artin. On the solutions of analytic equations. Invent. Math., 5:277-291, 1968.

[BE12] Carles Bivià-Ausina and Santiago Encinas. Łojasiewicz exponent of families of ideals, Rees mixed multiplicities and Newton filtrations. ArXiv e-prints, http://arxiv.org/abs/1103.1731v2:1-25, Jan 2012. 
[Biv09] Carles Bivià-Ausina. Local Łojasiewicz exponents, Milnor numbers and mixed multiplicities of ideals. Math. Z., 262(2):389-409, 2009.

[BKO12] Szymon Brzostowski, Tadeusz Krasiński and Grzegorz Oleksik. A conjecture on the Łojasiewicz exponent. J. Singul., 6:124-130, 2012. Singularities in Geometry and Appl., Będlewo (2011).

[CK88] Jacek Chądzyński and Tadeusz Krasiński. The Łojasiewicz exponent of an analytic mapping of two complex variables at an isolated zero. In Singularities (Warsaw, 1985), volume 20 of Banach Center Publ., pages 139-146. PWN, Warsaw, 1988.

[CK95] Jacek Chądzyński and Tadeusz Krasiński. Resultant and the Łojasiewicz exponent. Ann. Polon. Math., 61(1):95-100, 1995.

[Gre86] Gert-Martin Greuel. Constant Milnor number implies constant multiplicity for quasihomogeneous singularities. Manuscripta Math., 56(2):159-166, 1986.

[KL77] Tzee Char Kuo and Yung Chen Lu. On analytic function germs of two complex variables. Topology, 16(4):299-310, 1977.

[KOP09] Tadeusz Krasiński, Grzegorz Oleksik and Arkadiusz Płoski. The Łojasiewicz exponent of an isolated weighted homogeneous surface singularity. Proc. Amer. Math. Soc., 137(10):3387-3397, 2009.

[Len98] Andrzej Lenarcik. On the Łojasiewicz exponent of the gradient of a holomorphic function. In Singularities Symposium - Łojasiewicz 70 (Kraków, 1996; Warsaw, 1996), volume 44 of Banach Center Publ., pages 149-166. Polish Acad. Sci., Warsaw, 1998.

[LT08] Monique Lejeune-Jalabert and Bernard Teissier. Clôture intégrale des idéaux et équisingularité. Ann. Fac. Sci. Toulouse Math. (6), 17(4):781-859, 2008.

[O'S87] Donal B. O'Shea. Topologically trivial deformations of isolated quasihomogeneous hypersurface singularities are equimultiple. Proc. Amer. Math. Soc., 101(2):260-262, 1987.

[Ole09] Grzegorz Oleksik. The Łojasiewicz exponent of nondegenerate singularities. Univ. Iagel. Acta Math., 47:301-308, 2009.

[Ole13] Grzegorz Oleksik. The Łojasiewicz exponent of nondegenerate surface singularities. Acta Math. Hungar., 138(1-2):179-199, 2013.

[PT12] Camille Plenat and David Trotman. On the multiplicities of families of complex hypersurface-germs. ArXiv e-prints, http://arxiv.org/abs/1202.5177v5:1-8, Jun 2012.

[Pło85] Arkadiusz Płoski. Sur l'exposant d'une application analytique. II. Bull. Polish Acad. Sci. Math., 33(3-4):123-127, 1985.

[Pło88] Arkadiusz Płoski. Multiplicity and the Łojasiewicz exponent. In Singularities (Warsaw, 1985), volume 20 of Banach Center Publ., pages 353-364. PWN, Warsaw, 1988.

[Pło01] Arkadiusz Płoski. On the maximal polar quotient of an analytic plane curve. Kodai Math. J., 24(1):120$133,2001$.

[Pło10] Arkadiusz Płoski. Semicontinuity of the Łojasiewicz exponent. Univ. Iagel. Acta Math., 48:103-110, 2010.

[Sai71] Kyoji Saito. Quasihomogene isolierte Singularitäten von Hyperflächen. Invent. Math., 14:123-142, 1971.

[Sęk10] Maciej Sękalski. Note on the Łojasiewicz exponent of weighted homogeneous isolated singularities. Univ. Iagel. Acta Math., 48:133-136, 2010.

[Tei77] Bernard Teissier. Variétés polaires. I. Invariants polaires des singularités d'hypersurfaces. Invent. Math., 40(3):267-292, 1977. 
[Tro80] David Trotman. Équisingularité et conditions de Whitney. Thèse d'État, Université de Paris-Sud, Orsay, 1980.

[TYZ10] Shengli Tan, Stephen S.-T. Yau and Huaiqing Zuo. Łojasiewicz inequality for weighted homogeneous polynomial with isolated singularity. Proc. Amer. Math. Soc., 138(11):3975-3984, 2010.

[Var82] Aleksandr Nikolaevich Varchenko. A lower bound for the codimension of the $\mu=$ const stratum in terms of the mixed Hodge structure. Vestnik Moskov. Univ. Ser. I. Mat. Mekh., 6:28-31, 1982.

Szymon Brzostowski Faculty of Mathematics and Computer Science

University of Łódź ul. Banacha 22,

90-238 Łódź, Poland

brzosts@math.uni.lodz.pl 